\title{
CONTRIBUCIÓN AL PLAN DE GASIFICACIÓN DE ESPAÑA
}

\author{
Luis María Rodríguez González, Ing. Industrial
}

Subdirector de Ingeniería y Tecnología de ENAGAS (Empresa Nacional del Gas)

\section{INTRODUCCION}

\section{Plan de gasificación de España}

España, en buena medida, ha basado su desarrollo económico en la utilización del petróleo, por lo que el papel jugado hasta la fecha por el gas natural ha sido modesto. Es bien conocido que el consumo de gas natural se inicia en 1969, pero no es hasta mediados de 1980 cuando comienza el transporte y distribución de gas natural por distintas regiones de la geografía nacional.

Las incertidumbres existentes sobre la política de precios que iban a aplicar los países exportadores de petróleo y, en particular, Argelia, como principal suministrador, a raíz de la crisis energética de 1979, condujo a una indefinición de la política gasista que frenó el desarrollo de la infraestructura mínima imprescindible para absorber las cantidades contratadas. Sólo en febrero de 1985 se alcanza un acuerdo a nivel de los gobiernos de España y Argelia en materia de suministro de gas natural, siendo esta fecha el punto de partida de un ambicioso Plan de Gasificación a nivel nacional.

Este acuerdo tiene la importante virtud de ajustar las cantidades a importar de Argelia al previsible ritmo de crecimiento de la demanda que se derivará de la creación de la infraestructura gasista que incluye el Plan de Gasificación, y lo hace compatible con la producción anual esperada de los yacimientos nacionales a corto y medio plazo y con una estrategia de diversificación de los aprovisionamientos a largo plazo.

Despejada la incógnita del nivel de aprovisionamientos, la Administración ha promovido un acuerdo sectorial, suscrito en julio de 1985 por las distintas compañías distribuidoras y ENAGAS, que representa una intención de compromiso de todas las partes para desarrollar las acciones precisas que permitan cumplir los grandes objetivos del Plan, con 1992 como año horizonte.

Estos objetivos pueden resumirse en dos grupos diferentes, unos generales y otros especificos. Los primeros se concretan en la diversificación energética, aprovechamiento de los recursos nacionales, mejora del medio ambiente y ahorro energético; y los específicos contemplan un conjunto de acciones para duplicar la infraestructura del transporte y distribución, construir dos nuevas terminales de recepción de $\mathrm{GNL}^{*}$, desarro-
Ignacio Martínez Díaz, Ing. de Caminos

Jefe de Construcción de Nuevas Plantas de ENAGAS

(Empresa Nacional del Gas)

llar almacenamientos subterráneos y maximizar la producción de gas nacional.

En términos energéticos, el Plan de Gasificación se propone duplicar el consumo de gas natural en el período 1985-1992, incrementando su participación en el balance de energía primaria desde una cifra inferior al $3 \%$ en 1985, hasta un $6 \%$ en el año horizonte.

El esfuerzo necesario para cumplir el Plan es un importante reto para el sector gasista. Su ejecución implica la realización de un conjunto de actividades que tiene un impacto notable en la generación de empleo, tanto de carácter temporal, durante el período en el que deben desarrollarse las inversiones, como de carácter fijo, para la operación y mantenimiento de las nuevas instalaciones así como para la intensa gestión comercial que será preciso realizar.

Dentro de estas actividades merece destacarse la realización del esquema de infraestructura del Suroeste, que tiene como principal elemento al Terminal de GNL de Palos de la Frontera.

\section{Zona de influencia de la planta de regasificación de Huelva. Ampliación}

El sistema de gas natural, de la zona Suroeste, es en principio autónomo y consta de una Estación de $\mathrm{Ca}$ becera de Línea para la importación de Gas Natural Li. cuado (GNL) Gasoducto Huelva-Sevilla y Redes de distribución de Huelva y Sevilla.

La Estación de Cabecera de Línea para la importación de GNL se compone de una Planta de recepción, almacenamiento y regasificación de GNL procedente de Argelia, con una capacidad de almacenamiento de $60.000 \mathrm{~m}^{3}$ de GNL y una capacidad máxima de regasificación de $120.000 \mathrm{~m}^{3}(\mathrm{n}) / \mathrm{h}$, situada en terrenos de Puerto Autónomo de Huelva, en el término municipal de Palos de la Frontera.

De dicha estación de cabecera se alimentan el gasoducto de transporte Huelva-Sevilla con una longitud de $89 \mathrm{~km}$ y un diámetro de 20", diseñado para operar a una presión de 72 bar, y la red de distribución de Huelva, con una longitud de $26 \mathrm{~km}$ en diámetros comprendi-

${ }^{\star}$ GNL = Gas Natural Licuado. 
dos entre 2" y 14" y diseñada para operar a una presión de 16 bar.

La red de Sevilla se alimenta del gasoducto HuelvaSevilla y comprende el tendido de $81 \mathrm{~km}$ de red en diámetros entre 2" y 16 " y diseñada también para operar a 16 bar.

Esta infraestructura permitirá abastecer a las principales industrias del Polo de Huelva y el cinturón industrial de Sevilla -Dos Hermanas-Alcalá de Guadaira, así como a los usuarios domésticos y comerciales situados en zona de influencia, cifrándose el consumo potencial en casi 6.000 millones de termias correspondiendo el $94 \%$ a usuarios industriales y el resto a domésticos y comerciales que alcanzarán los 90.500 abonados.

Las instalaciones de la Planta de regasificación de $\mathrm{Pa}$ los de la Frontera permiten la ampliación en el futuro de las mismas con la construcción de un segundo depósito de almacenamiento y la adecuación de los equipos de bombas, compresores y vaporizadores, posibilitando el suministro de gas natural a otras zonas de influencia más alejadas, como la zona Centro mediante la conexión del gasoducto Huelva-Sevilla con Madrid, proyecto actualmente en fase de estudio.

\section{Consideraciones técnicas y económicas sobre el transporte y almacenamiento del gas natural}

El gas natural se extrae de yacimientos subterráneos dispersos por toda la geografía de la Tierra.

La mayor parte de los centros importantes de consumo se encuentran en lugares distantes de los centros de producción, por lo que se hace necesario transpor-

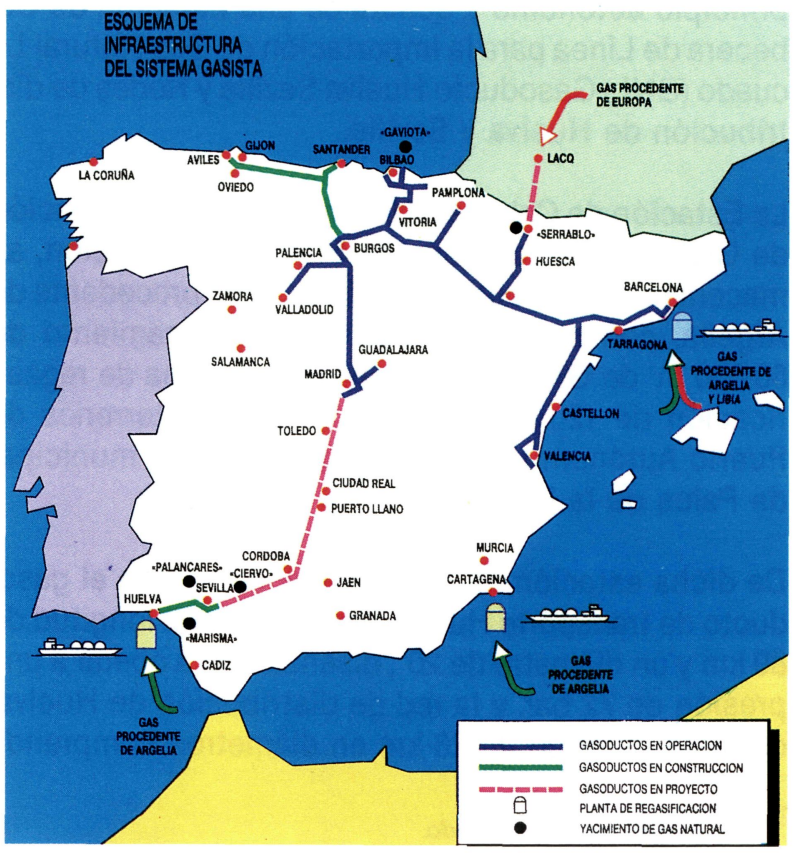

(C) Consejo Superior de Investigaciones Científicas Licencia Creative Commons 3.0 España (by-nc) tar el gas a grandes distancias para a continuación distribuirlo a los consumidores de esta energía.

El transporte a largas distancias se puede hacer por distintos métodos. El más extendido es por tuberías de gran diámetro, que trabajan a presiones entre 70 y 100 bar (gasoductos). También se puede transportar en recipientes a gran presión (250-300 bar), o bien transformando el gas natural en metanol para así efectuar el transporte como líquido. Finalmente otro método bastante utilizado consiste en licuar el gas natural (GNL) con lo que se consigue una disminución del volumen a transportar de unas 600 veces.

La selección del procedimiento de transporte en cada caso concreto se decide, como es lógico, por motivos económicos o por condicionantes técnicos.

El procedimiento más extendido en el transporte internacional (a nivel mundial) es por tubería con un $70 \%$ del total del gas transportado, seguido de las cadenas de licuación (GNL) con un $23 \%$ del tráfico.

El transporte del gas natural comprimido o transformado en metanol carece de entidad por su mayor coste.

Aunque un estudio comparativo, con sus ventajas técnicas y económicas, entre los dos principales sistemas de transporte de gas natural requeriria una exposición más detallada, conviene mencionar como idea general que para distancias menores de $4.000 \mathrm{~km}$ es más barato el transporte por gasoducto y para distancias superiores se debe considerar la cadena de GNL. En determinadas ocasiones y por imperativos técnicos o políticos, como el tener que cruzar mares profundos, es necesario transportar el gas natural como GNL.

Una cadena de transporte de Gas Natural Licuado (GNL) comprende una planta de licuación y un puerto de carga de metaneros situados en el país exportador, un puerto de recepción de metaneros y un terminal metanero para el almacenamiento y la regasificación del GNL en el país importador.

Actualmente existen en el mundo 17 cadenas en operación y 2 en construcción.

El futuro de las cadenas actuales de producción y transporte de gas natural licuado (GNL) parece garantizado, así como a medio-largo plazo la creación de otras nuevas, debido a que la distribución geográfica de los yacimientos de gas es muy diferente de la de los países consumidores.

La licuación del gas natural se efectúa a partir de un ciclo, bien sea de cascada clásica utilizando turbocompresores centrífugos de aspiración fría propano-etilenometano, bien sea mediante la utilización de una cascada incorporada con un fluido criogénico único y un solo compresor. 
Para el transporte marítimo, es necesario señalar que la débil densidad del metano líquido $(0,4)$ necesita dimensiones importantes para transportar el mismo tonelaje útil con relación a un petrolero.

El principal problema de la construcción de los metaneros es el de los depósitos, ya que es preciso dar respuesta a los problemas planteados tanto por las bajas temperaturas como por los esfuerzos debidos al movimiento del barco en el mar.

Los metaneros actualmente en servicio o en curso de construcción se reparten en dos grandes familias:

- Depósitos autoportantes y

- Depósitos integrados.

En estos últimos, las funciones de resistencia y estanquidad son distintas: la función resistente es asegurada por las estructuras mismas del barco; la estanquidad se realiza por una membrana delgada solidaria con la instalación y que trasmite al casco, en forma de presiones, los esfuerzos debidos a la carga.

En el terminal de recepción, la regasificación no plantea mayores problemas, y solamente el almacenamiento de gas natural en estado líquido necesita precauciones especiales. Los tanques de almacenamiento de GNL más utilizados en el mundo son los depósitos aéreos metálicos de doble pared. Algunos tanques tienen una tercera pared exterior realizada en hormigón pretensado, bien sean tanques aéreos o enterrados. En la actualidad se utilizan preferentemente los depósitos aéreos de doble contención con un depósito interior metálico (acero inoxidable o acero $9 \% \mathrm{Ni}$ ) y un depósito exterior realizado en hormigón pretensado.

Generalmente, la capacidad de los tanques es del orden de vez y media a dos veces la capacidad de los bar$\cos$, si bien se tiende, sobre todo en los puertos de recepción, a aumentar la capacidad de almacenamiento así como la potencia de regasificación, a fin de poder suministrar un caudal suplementario para asegurar las puntas de consumo.

\section{Dimensionamiento y concepción del Tanque de GNL de Huelva}

El tanque de almacenamiento de Huelva tiene una capacidad neta de $60.000 \mathrm{~m}^{3}$ de GNL en estado cercano a su punto de ebullición, presión atmosférica y $-163^{\circ} \mathrm{C}$.

Su concepción, de acuerdo con las últimas innovaciones habidas en el diseño de este tipo de depósitos, responde a estrictos requerimientos de seguridad y fiabilidad.

Para poder mantener el gas en el depósito en estado líquido y a baja temperatura, el tanque se compone de tres elementos principales: a) El recipiente interno, destinado a contener el gas licuado a temperatura criogénica, totalmente aislado y separado por este aislamiento de cualquier contacto con el ambiente exterior. Este contenedor primario está constituido por chapa de acero aleado al $9 \% \mathrm{Ni}$, con espesor decreciente desde el fondo hacia arriba para asegurar una tasa de trabajo del metal sensiblemente constante.

b) El aislante, que rodea totalmente el recipiente interno o contenedor primario, y evita el aporte de calorias del exterior al GNL, disminuyendo por tanto la evaporación.

c) El recipiente externo, que sirve para contener el aislante antes descrito, y es estanco a los vapores de GNL, estando normalmente a temperatura ambiente. Este recipiente incorpora funciones de contenedor secundario en caso de rotura del tanque interior, y se proyecta con fuertes requisitos de seguridad incorporando a las funciones de contenedor secundario las de protección frente a derrames de GNL procedentes de depósitos adyacentes, radiaciones térmicas debidas a fuego externo o interno, explosiones o impactos de elementos de sabotaje, aviación, etc.

Actualmente la tendencia en la construcción de tanques responde al concepto de doble integridad, es decir, un tanque exterior de hormigón pretensado capaz de retener el GNL procedente de una eventual fuga en el tanque interior, constituyendo así un cubeto adosado a dicho tanque interior. Además se incorporan a su diseño los siguientes condicionantes que confieren al mismo una especial seguridad:

- Todas las penetraciones al tanque se realizan por la cúpula, sin entradas por el fondo o cavidades laterales.

- Resistir sin colapsar durante el tiempo que tardaría en arder el GNL contenido en el tanque.

- Resistir en condición operativa a la radiación de un fuego exterior que aportase una densidad energética de $12 \mathrm{kw} / \mathrm{m}^{2}$ durante 6 horas.

- Resistir al impacto de un sólido similar a un avión pequeño.

- Redundancia del sistema de vigilancia de nivel con prealarma, alarma y parada de la descarga del metanero por muy alto nivel.

- Triple seguridad ante vacío, con un primer escalón de entrada de gas natural, segundo de nitrógeno y tercero de entrada de aire por válvula rompedora de vacío.

- Vigilancia continua de la variación de la densidad con la profundidad para detectar una eventual estratificación y proceder a homogeneizar el contenido del tanque a fin de evitar fenómenos de "roll over".

http://informesdelaconstruccion.revistas.csic.es 
La entrada de GNL, procedente de los buques metaneros, se efectúa normalmente por el techo del tanque existiendo la posibilidad de inyectar el GNL hasta el fondo, o en la parte alta del tanque, en función de la densidad del producto de la presión de almacenamiento en el barco, con el fin de evitar el riesgo de estratificación atípica de capas de GNL.

\section{Puesta en marcha y explotación}

El tanque de GNL ha de ser probado hidrostática y neumáticamente antes de que las bombas de emisión de GNL de baja presión sean instaladas en el interior del mismo.

La prueba hidráulica del tanque podrá hacerse con agua hasta una altura equivalente al peso de GNL cuando el tanque se encuentre completamente lleno.

Realizadas satisfactoriamente las pruebas hidrostática y neumática, el tanque debe ser purgado y secado, llevando el punto de rocío a valores del orden de $-25^{\circ} \mathrm{C}$ en el depósito interior, y valores algo mayores, del orden de $-15^{\circ} \mathrm{C}$, en el espacio de aislamiento entre el tanque interior y exterior. Asimismo, el contenido en oxígeno debe ser inferior al $8 \%$ en volumen. Estas operaciones se realizan mediante la inyección de nitrógeno en el interior del tanque.

Antes de la primera descarga de GNL, el tanque debe ser previamente enfriado con nitrógeno líquido de una manera lenta, asegurando un gradiente adecuado de temperaturas para evitar tensiones excesivas en el depósito interior.

Por otro lado, el sistema rompedor de vacío debe ser operativo antes de introducir el GNL en el tanque.

La descarga del primer barco debe hacerse con suma precaución. El caudal de GNL al tanque debe enviarse a las líneas de llenado por el fondo. El nitrógeno se desplazará hacia la parte superior hasta el sistema de venteo.

El nitrógeno desplazado del tanque de almacenamiento irá al sistema de antorcha a través de las líneas de vapor del tanque.

Cuando el barco se ha descargado y con los niveles ya establecidos de GNL en el tanque de almacenamiento, el terminal está preparado para la operación normal.

El depósito se diseña para una presión diferencial con la atmosférica comprendida entre $+290 \mathrm{~m}$ bar y $-10 \mathrm{~m}$ bar. Las presiones de operación normal (sin alarmas) oscilan entre un mínimo de $100 \mathrm{~m}$ bar y un máximo de
$250 \mathrm{~m}$ bar. A $255 \mathrm{~m}$ bar se produce la alarma por alta presión. A $260 \mathrm{~m}$ bar se produce la alarma por muy alta presión y además se cierra automáticamente la entrada de GNL procedente del metanero. A $265 \mathrm{~m}$ bar alivian las válvulas de seguridad que descargan a la antorcha (primer escalón). A $285 \mathrm{~m}$ bar abre el segundo escalón de válvulas que descargan a la antorcha y a $290 \mathrm{~m}$ bar la descarga a la atmósfera. La alarma por baja presión se produce a $25 \mathrm{~m}$ bar. A $15 \mathrm{~m}$ bar se produce la alarma de muy baja presión, y, como acción correctora, el disparo de las bombas primarias, simultáneamente con la aportación de gas natural desde el gasoducto. A los $10 \mathrm{~m}$ bar entra nitrógeno desde la red de la planta y $\mathrm{a}-5 \mathrm{~m}$ bar se abren las válvulas rompedoras de vacío que admiten aire atmosférico.

Durante la operación del Terminal el tanque de GNL se encuentra permanentemente vigilado a través de las conexiones de instrumentación asociadas a la medición de temperaturas, presión, nivel, densidad, contenido en oxígeno y toma de muestras.

La temperatura de los elementos estructurales del tanque se mide por instalación de termopares en paredes y fondo, que se utilizan esencialmente durante la puesta en frío para conseguir velocidades de descenso de temperatura y gradientes aceptables.

La temperatura del GNL se mide de dos formas. La primera es combinada con la medición de nivel y densidad por medio de una sonda móvil que se traslada a lo largo de la altura del tanque. Estas mediciones de temperatura y densidad en función del nivel sirven para detectar estratificación. La segunda forma de medición de temperatura es por termorresistencias ubicadas en el líquido a diferentes alturas. También se mide la temperatura del espacio vapor bajo el techo suspendido. La presión en el espacio vapor bajo el techo suspendido se mide por medio de un transmisor de presión absoluta y otro de presión manométrica.

Además de la medición de nivel que se deduce de las lecturas de temperatura y densidad a diferentes alturas, se instalan dos instrumentos indicadores de nivel, por palpador, alojados en tubo tranquilizador, que da las alarmas por alto y muy alto nivel y provoca el corte de entrada del GNL durante la descarga del metanero, para evitar el rebose del tanque interior.

Tanto para asegurar en la puesta en marcha la completa inertización del tanque, como con el mismo fin en operación (si llegara a producirse la entrada de aire por las rompedoras de vacio), existe una toma de muestra del espacio vapor conducida en continuo a un analizador de oxígeno. También existe una conexión de toma de muestra del gas contenido en el espacio anular, que permite analizar su composición. 